\title{
Safe discharge from the cardiac emergency room with a rapid rule-out myocardial infarction protocol using serial $\mathrm{CK}-\mathrm{MB}_{\text {mass }}$
}

\author{
R Bholasingh, R J de Winter, J C Fischer, R W Koster, R J G Peters, G T Sanders
}

\begin{abstract}
Objective-To determine whether a new protocol, using a rapid and sensitive $C K-M_{\text {mass }}$ assay and serial sampling, can rule out myocardial infarction in patients with chest pain and decrease their length of stay in the cardiac emergency room without increasing risk.

Design-The combined incidence of cardiac death and acute myocardial infarction at 30 days, six months, and 24 months of follow up were compared between patients discharged home from the cardiac emergency room after ruling out myocardial infarction with a $\mathrm{CK}-\mathrm{MB}_{\text {activity }}$ assay in 1994 and those discharged home after a rapid CK-MB mass $_{\text {assay in }} 1996$.

Setting-Cardiac emergency room of a large university hospital.

Patients-In 1994 and 1996, 230 and 423 chest pain patients, respectively, were discharged home from the cardiac emergency room with a normal CK-MB and an uneventful observation period.

Results-The median length of stay in the cardiac emergency room was significantly reduced, from 16.0 hours in 1994 to 9.0 hours in 1996 ( $\mathrm{p}<0.0001$ ). Mean event rates in patients from the 1994 and 1996 cohorts, respectively, were $0.9 \%$ (95\% confidence interval (CI) $-0.3 \%$ to $2.1 \%$ ) v $0.7 \%(95 \% \mathrm{CI}-0.1 \%$ to $1.5 \%)$ at 30 days, $3.0 \%$ (95\% CI $0.8 \%$ to $5.2 \%)$ v $2.8 \%(95 \% \mathrm{CI}$ $1.2 \%$ to $4.4 \%$ ) at six months, and $7.0 \%$ (95\% CI $3.7 \%$ to $10.3 \%)$ v $5.7 \%(95 \%$ CI $3.5 \%$ to $7.9 \%)$ at 24 months. Kaplan-Meier survival analysis showed no difference in mean event-free survival at 30 days, six months, and 24 months of follow up.

Conclusions-Using a rule-out myocardial infarction protocol with a rapid and sensitive $\mathrm{CK}-\mathrm{MB}_{\text {mass }}$ assay and serial sampling, the length of stay of patients with chest pain in the cardiac emergency room can be reduced without compromising safety.

(Heart 2001;85:143-148)
\end{abstract}

Keywords: length of stay; cardiac emergency room; creatine kinase-MB; myocardial infarction

It is important to rule out acute myocardial infarction early in the triage of patients presenting with chest pain. Patients at low risk of a cardiac event ${ }^{1}$ can then be discharged home safely, while those at intermediate or high risk should be admitted for further management.

Several biochemical markers can be used to rule out myocardial infarction in the early stages. These are cardiac troponins, ${ }^{2-5}$ myoglobin, ${ }^{346}$ and the creatine kinase $\mathrm{MB}$ isoenzyme $(\mathrm{CK}-\mathrm{MB}) .^{3478}$ In a recent study by Zimmerman and colleagues, ${ }^{3}$ although the most sensitive early marker of myocardial infarction was the analysis of CK-MB subforms $(91 \%$ at six hours after the onset of symptoms), CK-MB $\mathrm{Bass}_{\text {mas }}$ used as the diagnostic standard "because it has been the diagnostic standard worldwide for more than two decades and because extensive clinical and experimental evidence indicates increased plasma CK-MB reflects infarction." Hamm and colleagues recently reported that cardiac troponins may also be effective for early triage, ${ }^{9}$ and they showed that patients with chest pain in the emergency room are at low risk of subsequent cardiac events after negative (serial) troponin $\mathrm{T}$ or I test results.

It has been found that the sensitivity and specificity of cardiac markers for the early diagnosis of myocardial infarction change rap- idly in the first six hours after the onset of symptoms, ${ }^{34}$ so serial measurements may increase the sensitivity of these tests for detecting myocardial infarction. ${ }^{4}$ Rule-out protocols have most often relied on serial CK-MB measurements. ${ }^{78^{10-12}}$ With the development of a new, rapid, and sensitive $C K-M B_{\text {mass }}$ assay, early triage of patients presenting with chest pain is feasible and may result in a reduction in the length of stay in the cardiac emergency room.

In the present study, we evaluated prospectively a new rule-out myocardial infarction protocol which was introduced into our cardiac emergency room in 1995. This replaced the $\mathrm{CK}-\mathrm{MB}_{\text {activity }}$ assay with a rapid and sensitive $\mathrm{CK}-\mathrm{MB}_{\text {mass }}$ assay and serial sampling. Our aim in this study was to determine whether this protocol decreased the length of stay in the cardiac emergency room while preserving the overall risk assessment.

\section{Methods}

To assess the efficacy and safety of the new rule-out myocardial infarction protocol, we designed a prospective 30 days, six months, and 24 months follow up study of all patients who were discharged home from our cardiac emergency room during 1994 and 1996. 
PATIENTS

Patients presenting with chest pain to our cardiac emergency room usually stay for observation to exclude severe instability and to rule out myocardial infarction before they are discharged home. Our cardiac emergency room is a five bed department equipped with ECG monitoring, where patients can remain under observation for a maximum of 24 hours. In 1995, a new rule-out myocardial infarction protocol using serial $\mathrm{CK}-\mathrm{MB}_{\text {mass }}$ was introduced.

In 1994, blood samples were routinely drawn at admission and at $6,12,18$, and 24 hours after the onset of symptoms to measure $\mathrm{CK}-\mathrm{MB}_{\text {activity. }}$ The results were made available twice daily: at 11.00 and 16.00 hours.

In 1996, blood samples were drawn on admission and at 5, 7, and 10 hours after the onset of symptoms to measure CK-MB mass $_{\text {. The }}$ results were made available within one hour from blood sampling, the last result being available 11 hours after the onset of symptoms.

Patients with chest pain were included in the present study when they were discharged home from the cardiac emergency room after normal CK-MB measurements and an uneventful observation period. These patients were followed up. Patients with abnormal or nondiagnostic ECGs were included in both cohorts. Patients were excluded if they were transferred to the coronary care unit. Patients were transferred to the coronary care unit for the following reasons: if there was evidence of minor myocardial damage or myocardial inf$\operatorname{arction}^{13}{ }^{14}$; if there was unstable angina defined as recurrent symptoms together with concomitant dynamic ECG changes; or on the basis of the clinical judgement of the attending physician.

For patients who presented more than once to the cardiac emergency room, only the first admission was included.

An exercise tolerance test was not routinely performed in all patients before discharge home from the cardiac emergency room but was available at the request of the attending physician. In addition, on discharge the patients usually received drug treatment and an appointment for the outpatient clinic, at the discretion of the attending physician.

The study was approved by the locally appointed ethics committee and informed consent was obtained.

ASSAYS

1994 Cohort

$\mathrm{CK}-\mathrm{MB}_{\text {activity }}$ was determined using an ion exchange column chromatography method, the CK-MM and CK-MB fractions being separated on a Sephadex A-50 column. After separation, the activities were measured with a commercial kit (CPK, acetylcysteine activated, product 124184, Boehringer Mannheim, Germany). The reaction was started by the addition of creatine phosphate and the activities were measured at $340 \mathrm{~nm}$ in a spectrophotometer. ${ }^{15} 16$ The upper limit for myocardial infarction was $8.0 \mathrm{U} / 1$. Precision, as expressed
Table 1 Baseline characteristics of the 1994 and 1996 cohorts

\begin{tabular}{lll}
\hline & 1994 Cohort & 1996 Cohort \\
\hline Total & 230 & 423 \\
Male & $127(55)$ & $244(58)$ \\
Age (years) & $63(24$ to 94$)$ & $61(27$ to 96$)$ \\
Diabetes mellitus & $17(7)$ & $65(15)^{\star}$ \\
Hypertension & $48(21)$ & $125(30) \dagger$ \\
Smoking & $62(27)$ & $122(29)$ \\
Previous MI & $83(36)$ & $103(24) \ddagger$ \\
Previous PTCA/CABG & $49(21)$ & $80(19)$ \\
Aspirin treatment & $67(29)$ & $144(34)$ \\
\hline
\end{tabular}

Values are $\mathrm{n}(\%)$ or (range).

${ }^{\star} \mathrm{p}=0.004 ; \mathrm{tp}=0.02 ; \neq \mathrm{p}=0.001$.

CABG, coronary artery bypass grafting; $\mathrm{MI}$, acute myocardial infarction; PTCA, percutaneous transluminal coronary angioplasty.

by the coefficient of variation, was $13.5 \%$ at the level of $6.31 \mathrm{U} / 1$. Assay temperature was $37^{\circ} \mathrm{C}$.

1996 Cohort

$\mathrm{CK}-\mathrm{MB}_{\text {mass }}$ was measured with the Immuno-1 analyser (Bayer, Leverkusen, Germany). The upper limit for myocardial infarction was $7.0 \mu \mathrm{g} / \mathrm{l}$. Precision (coefficient of variation) was $2.5 \%$ at the level of $5.0 \mu \mathrm{g} / 1$. Assay temperature was $37^{\circ} \mathrm{C}$. An increase in serial $\mathrm{CK}-\mathrm{MB}_{\text {mass }}$ values larger than the reference change value $(2.0 \mu \mathrm{g} / \mathrm{l})$ was considered abnormal. ${ }^{13}$

Myocardial infarction was defined according to the World Health Organization criteria. ${ }^{14}$ Minor myocardial damage was defined either as a typical rise and fall of $\mathrm{CK}-\mathrm{MB}_{\text {mass }}$ with a peak about the upper limit of normal but less than or equal to twice the upper limit of normal, or as a significant increase in serial $C K-M B_{\text {mass }}$ values of more than the reference change value. $^{13} 17$

FOLLOW UP

The 30 days, six months, and 24 months follow up assessments were made by telephone interview with either the patient, the patient's relatives, the cardiologist, or the patient's general practitioner. In case of a cardiac event, either the medical records were reviewed or the patient's general practitioner or cardiologist was consulted to confirm the diagnosis. A cardiac event was defined as non-fatal myocardial infarction or as death from cardiac causes (fatal myocardial infarction, sudden cardiac death, or death from other cardiac causes), which occurred after discharge from the cardiac emergency room.

STATISTICAL ANALYSIS

Comparisons of frequency distribution between groups were performed with the $\chi^{2}$ test. To assess event-free survival, Kaplan-Meier curves were constructed for the cardiac events, and differences in mean survival were compared using the log-rank test. Difference between median lengths of stay was assessed by the Mann-Whitney U test.

\section{Results}

In 1994,1892 patients presented at the cardiac emergency room. Of these, 1109 (59\%) had chest pain and 393 (35\%) were admitted to the coronary care unit. The other patients with chest pain were either discharged from the car- 
Table 2 Cardiac events (cardiac death and non-fatal acute myocardial infarction) in the 1994 and 1996 cohorts during 30 days, 6 months, and 24 months of follow up

\begin{tabular}{llllc}
\hline \multirow{2}{*}{ Cohort } & $\begin{array}{l}\text { Cardiac } \\
\text { event }\end{array}$ & $\begin{array}{l}\text { Follow up: } \\
\text { 30 days }\end{array}$ & $\begin{array}{l}\text { Follow up: } \\
\text { 6 months }\end{array}$ & $\begin{array}{l}\text { Follow up: } \\
\text { 24 months }\end{array}$ \\
\hline 1994 (n=230) & CD & 1 & 3 & 7 \\
& MI & 1 & 4 & 9 \\
& Total & 2 & 7 & 16 \\
$1996(\mathrm{n}=423)$ & CD & 1 & 6 & 9 \\
& MI & 2 & 6 & 15 \\
& Total & 3 & 12 & 24 \\
\hline
\end{tabular}

$\mathrm{CD}$, cardiac death; MI, acute myocardial infarction.

diac emergency room without CK-MB measurements because of the diagnosis of atypical or non-cardiac chest pain (486 of 1109 (44\%)), or they were included in the rule-out myocardial infarction protocol and remained under observation at the cardiac emergency room (230 of $1109(21 \%))$.

In 1996, 2266 patients presented at the cardiac emergency room. Of these, $1162(51 \%)$ had chest pain and 398 (34\%) were admitted to the coronary care unit. The other patients with chest pain were either discharged from the cardiac emergency room without CK-MB measurements because of the diagnosis atypical or non-cardiac chest pain (341 of 1162
$(29 \%))$, or they were included in the rule-out myocardial infarction protocol and remained under observation at the cardiac emergency room (423 of $1162(36 \%)$ ).

Thus, according to the inclusion criteria, the 1994 cohort comprised 230 patients and the 1996 cohort comprised 423 patients. The baseline characteristics are shown in table 1 : diabetes mellitus and hypertension were more prevalent in the 1996 cohort $(15 \%$ v $7 \%$, respectively $(\mathrm{p}=0.004)$, and $30 \%$ v $21 \%$ $(\mathrm{p}=0.02))$, whereas a history of myocardial infarction was more prevalent in the 1994 cohort $(36 \%$ v $24 \%(\mathrm{p}=0.001))$. Other characteristics were not different between the two cohorts.

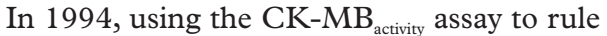
out myocardial infarction, the median length of stay in the cardiac emergency room was 16.0 hours (range 1.25-47.2 hours). In 1996, using the new, rapid, and sensitive $\mathrm{CK}-\mathrm{MB}_{\text {mass }}$ assay, the median length of stay in the cardiac emergency room was significantly reduced by $44 \%$ to a median of 9.0 hours (range 1.0-52.0 hours) $(\mathrm{p}<0.0001)$.

Follow up of the 1994 and the 1996 cohorts was, respectively, $95 \%$ and $99 \%$ complete. The cardiac events in both cohorts which occurred

Table 3 Cardiac events in the 1994 cohort $(n=16)$ : patient characteristics and days of follow up

\begin{tabular}{rllllllll}
\hline & & & & & & Previous & $\begin{array}{l}\text { Previous } \\
\text { CABG }\end{array}$ & $\begin{array}{l}\text { Risk factors (n) (DM, } \\
\text { smoking or hypertension) }\end{array}$ \\
\hline 1 & Sex & Age (years) & CE & FU (days) & Previous MI & PTCA & - & 0 \\
2 & M & 68 & MI & 8 & + & - & - & 2 \\
3 & M & 41 & CD & 15 & - & - & - & 1 \\
4 & F & 78 & MI & 41 & + & - & - & 1 \\
5 & M & 65 & MI & 77 & - & - & - & 1 \\
6 & M & 65 & MI & 90 & - & - & + & 1 \\
7 & M & 60 & CD & 177 & + & - & - & 1 \\
8 & F & 56 & MI & 205 & + & - & - & 1 \\
9 & M & 43 & MI & 218 & + & - & - & 0 \\
10 & M & 83 & CD & 240 & + & - & - & 0 \\
11 & F & 59 & MI & 282 & + & + & - & 0 \\
12 & M & 72 & MI & 317 & + & - & - & 1 \\
13 & M & 67 & CD & 439 & + & - & - & 0 \\
14 & F & 82 & CD & 462 & + & - & - & 0 \\
15 & F & 80 & MI & 515 & + & - & - & - \\
16 & M & 80 & CD & 643 & + & - & - & - \\
\hline
\end{tabular}

CABG, coronary artery bypass grafting; CD, cardiac death; CE, cardiac event; DM, diabetes mellitus; MI, acute myocardial infarction; PTCA, percutaneous transluminal coronary angioplasty; +, present; -, absent.

Table 4 Cardiac events in the 1996 cohort $(n=24)$ : patient characteristics and days of follow up

\begin{tabular}{|c|c|c|c|c|c|c|c|c|}
\hline & Sex & Age (years) & $C E$ & $\begin{array}{l}\text { Follow up } \\
\text { (days) }\end{array}$ & Previous MI & $\begin{array}{l}\text { Previous } \\
\text { PTCA }\end{array}$ & $\begin{array}{l}\text { Previous } \\
C A B G\end{array}$ & $\begin{array}{l}\text { Risk factors ( } n \text { ) (DM, } \\
\text { smoking or hypertension) }\end{array}$ \\
\hline 1 & $\mathrm{~F}$ & 93 & MI & 3 & + & - & - & 0 \\
\hline 2 & $M$ & 75 & MI & 10 & - & - & - & 0 \\
\hline 3 & $M$ & 76 & CD & 19 & - & - & - & 0 \\
\hline 4 & $M$ & 65 & $\mathrm{CD}$ & 62 & + & - & + & 0 \\
\hline 5 & M & 57 & MI & 65 & - & - & - & 2 \\
\hline 6 & M & 71 & MI & 66 & + & - & + & 2 \\
\hline 7 & $M$ & 73 & $\mathrm{CD}$ & 73 & + & - & - & 2 \\
\hline 8 & $M$ & 64 & CD & 79 & + & + & + & 1 \\
\hline 9 & $\mathrm{~F}$ & 82 & $\mathrm{CD}$ & 104 & - & - & - & 0 \\
\hline 10 & $\mathrm{~F}$ & 74 & MI & 113 & + & - & - & 3 \\
\hline 11 & $M$ & 66 & MI & 117 & - & - & _ & 1 \\
\hline 12 & $\mathrm{~F}$ & 79 & $\mathrm{CD}$ & 145 & - & - & - & 0 \\
\hline 13 & $\mathrm{~F}$ & 55 & MI & 182 & - & - & - & 1 \\
\hline 14 & $M$ & 59 & $\mathrm{CD}$ & 185 & - & - & - & 2 \\
\hline 15 & $M$ & 53 & MI & 226 & - & - & - & 3 \\
\hline 16 & $M$ & 47 & MI & 250 & - & - & - & 1 \\
\hline 17 & $\mathrm{~F}$ & 62 & MI & 274 & + & - & + & 1 \\
\hline 18 & $\mathrm{~F}$ & 96 & MI & 300 & - & - & - & 0 \\
\hline 19 & $\mathrm{~F}$ & 84 & $\mathrm{CD}$ & 315 & - & - & - & 0 \\
\hline 20 & $M$ & 79 & MI & 330 & + & - & + & 1 \\
\hline 21 & $M$ & 73 & MI & 455 & + & + & + & 1 \\
\hline 22 & $M$ & 67 & MI & 455 & + & - & + & 0 \\
\hline 23 & $M$ & 71 & MI & 519 & - & - & - & 3 \\
\hline 24 & $\mathrm{~F}$ & 74 & $\mathrm{CD}$ & 610 & + & - & - & 0 \\
\hline
\end{tabular}




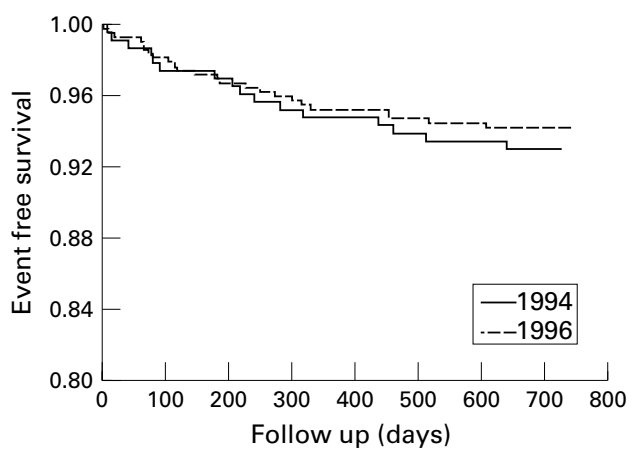

Figure 1 Kaplan-Meier survival curves during a follow up period of 24 months in patients from the 1994 and 1996 cohorts discharged home from the cardiac emergency room after normal $C K-M B$ results and an uneventful observation period. Event-free survival was assessed for cardiac death and non-fatal acute myocardial infarction. Differences were compared by the log-rank test. There was no significant difference in mean event-free survival between the two cohorts (at the 30 days follow up, $p=0.81$; at the six months follow up, $p=0.83$; at the 24 months follow $u p, p=0.56$ ).

during the follow up are shown in tables 2, 3, and 4 . At the 30 days follow up, two of 230 patients $(0.9 \%, 95 \%$ confidence interval (CI) $-0.3 \%$ to $2.1 \%$ ) from the 1994 cohort experienced an event, compared with three of 423 $(0.7 \%, 95 \%$ CI $-0.1 \%$ to $1.5 \%)$ from the 1996 cohort (NS). At the six months follow up, the event rates were $7 / 230(3.0 \%, 95 \%$ CI $0.8 \%$ to $5.2 \%)$ v $12 / 423(2.8 \%, 95 \%$ CI $1.2 \%$ to $4.4 \%)$ (NS). At the 24 months follow up, the event rates were $16 / 230(7.0 \%, 95 \%$ CI $3.7 \%$ to $10.3 \%)$ v $24 / 423(5.7 \%, 95 \%$ CI $3.5 \%$ to $7.9 \%$ ) (NS).

The Kaplan-Meier survival analysis (logrank test) showed no difference in mean eventfree survival between the two cohorts at the 30 days follow up $(p=0.81)$ (Kaplan-Meier survival curve not shown), at the six months follow up ( $p=0.83$ ), and at the 24 months follow up ( $\mathrm{p}=0.56)$ (fig 1$)$.

During the six months follow up, events occurred at a median of 77 days (range 8-177 days) in the 1994 cohort and at a median of 70 days (range 19-145 days) ( $p=0.9$ ) in the 1996 cohort, with an equal distribution over time.

Interestingly, in all patients who experienced a cardiac event during the entire follow up, coronary artery disease was either documented before the index cardiac emergency room admission or was established as the discharge diagnosis.

\section{Discussion}

Our data show that the application of a rule-out myocardial infarction protocol with a rapid and sensitive $C K-M B_{\text {mass }}$ assay and serial sampling can significantly reduce the length of stay of patients with chest pain in the cardiac emergency room without compromising safety. The incidence of events in patients discharged home from the cardiac emergency room was low during the 30 days, six months, and 24 months follow up.

Several triage protocols have been evaluated to identify low risk patients with chest pain ${ }^{118-27}$ and recommendations have been made. ${ }^{2528-30} \mathrm{To}$ identify low risk patients, it is as important to rule out myocardial infarction using cardiac markers as it is to exclude severe instability by means of an observation period in the cardiac emergency room. Early identification of low risk patients may result in a reduction in the length of stay in hospital (or in the cardiac emergency room). ${ }^{18}{ }^{31-34}$ Different cardiac marker test policies may affect the length of stay. The length of stay may be reduced significantly either when the results of serial measurements are made available in the morning as opposed to the end of the day, ${ }^{31}$ when an assay with a shorter turn around time is used, ${ }^{32}$ or when the testing policy is changed from batch to random access. ${ }^{33}$ Apple and colleagues compared a CK-MB $\mathrm{Bactivity}_{\text {assay }}$ with a rapid and sensitive $\mathrm{CK}-\mathrm{MB}_{\text {mass }}$ assay (Stratus, Dade Behring, USA) in a small study group (65 patients) admitted to a coronary care unit. $^{34}$ Results of CK-MB activity $_{\text {were }}$ made available once daily, whereas those of $\mathrm{CK}-\mathrm{MB}_{\text {mass }}$ were made available twice daily, and it was possible to obtain the result from a single specimen within 10 minutes. These investigators showed that the rapid and sensitive $\mathrm{CK}-\mathrm{MB}_{\text {mass }}$ assay improved triage and management of these patients, resulting in a reduction of between 3 and 23 hours in the length of stay in the coronary care unit and a reduction in costs compared with the $\mathrm{CK}-\mathrm{MB}_{\text {activity }}$ assay. Their general conclusions that a frequent and rapid testing policy, using a sensitive assay, is associated with a shorter length of stay in the hospital are in accordance with our results in the present study, which involved larger study cohorts.

Serial CK-MB measurements have been used for several years as the gold standard for the diagnosis of myocardial infarction. ${ }^{3142528} 29$ In addition, serial CK-MB measurements may be used effectively in early triage of patients with chest pain by ruling out myocardial infarction. $^{781013}$ Other cardiac markers that may be used as well for early triage are, for example, myoglobin 3635 and cardiac troponins. ${ }^{2-5} 9$ Myoglobin is an early marker ${ }^{4}$ but it is not heart specific. ${ }^{36}$ On the other hand, the troponins are heart specific and have the ability to detect minor myocardial damage, although the biological and analytic variation of the cardiac troponin I assays has not yet been established ${ }^{29}{ }^{30}$ and cardiac troponins are relatively late markers of myocardial infarction. ${ }^{3}$ However, for routine diagnosis of myocardial infarction an earlier marker has been recommended, such as $\mathrm{CK}-\mathrm{MB},{ }^{29}$ which has additional advantages of established biological and analytic characteristics ${ }^{30}$ and the ability to diagnose minor myocardial damage. ${ }^{7} 91013$

Polanczyk and colleagues evaluated a strategy for using $C K-M B_{\text {mass }}$ and troponin $I$ in patients presenting with chest pain. ${ }^{2}$ According to this strategy, patients were divided into four different risk groups for cardiac events occurring within 72 hours after admission. Positive troponin I values did not have additional value in low risk patients presenting with chest pain without evidence of acute ischaemia on an admission ECG and with normal CK-MB values (event rate $6 \%$ in the presence of positive troponin I $v 4 \%$ in presence of negative troponin I values). In addition, normal tro- 
ponin I values did not rule out the risk for a cardiac event during 72 hours after admission (event rate $4 \%$ ). These cardiac events occurred during the observation period up to 72 hours after admission. In the present study, these high risk patients were excluded; they were admitted to the coronary care unit for further evaluation and treatment. Furthermore, the event rate of $4 \%$ in the first 72 hours after admission is high compared with the 30 day event rate of $0.9 \%$ in the 1994 cohort and $0.7 \%$ in the 1996 cohort in the present study. This may reflect the definition used for cardiac events by Polanczyk and colleagues, ${ }^{2}$ which also included, for example, ventricular arrhythmias and revascularisation. These data on troponin I were recently confirmed by Johnson and colleagues using troponin $\mathrm{T}^{5}$.

In another study, with long term follow up, Ravkilde and associates evaluated the prognostic value of increased serum levels of the sensitive markers $C K-M B_{\text {mass }}$ and troponin $T$ in rule-out myocardial infarction patients. ${ }^{37} \mathrm{Myo}-$ cardial infarction was ruled out if serial CK and $\mathrm{CK}-\mathrm{MB}_{\text {activity }}$ values were normal during the sampling period of 48 hours. During a follow up of 28 months, 11 of 124 patients $(8.9 \%)$ without a myocardial infarct experienced a cardiac event. Dividing the rule-out myocardial infarction group into subgroups, the cardiac event rate was $3.4 \%$ in patients with negative $\mathrm{CK}-\mathrm{MB}_{\text {mass }}$ and $5.1 \%$ in those with negative troponin $\mathrm{T}$; these values are comparable with the 24 months event rates of $7.0 \%$ (95\% CI $3.7 \%$ to $10.3 \%$ ) in the 1994 cohort, and $5.7 \%$ (95\% CI $3.5 \%$ to $7.9 \%$ ) in the 1996 cohort, which we found in the present study.

Recently, cardiac troponins (troponin I and $\mathrm{T}$ ) have been shown to have a high sensitivity and specificity for detecting myocardial infarction and identifying patients at risk for a cardiac event. Hamm and colleagues reported that in patients presenting with acute chest pain in the emergency room, the incidence of events at 30 days was very low in those with a negative troponin $\mathrm{T}$ or I test: $1.1 \%(7 / 650)$ in patients with negative troponin $\mathrm{T}$ tests, and $0.3 \%(2 / 602)$ in those with negative troponin I tests. ${ }^{9}$ The follow up in this study was $97.2 \%$ complete. Cardiac events that occurred within the first 24 hours of hospital admission were excluded, and all patients-including those with a normal troponin result-were in fact admitted to the hospital. In our present study, the event rates at 30 days were comparable with the event rate in the study by Hamm and colleagues: $0.9 \%(2 / 230)$ in patients with a negative $\mathrm{CK}-\mathrm{MB}_{\text {activity }}$ test (1994 cohort), and $0.7 \%(3 / 423)$ in patients with a negative

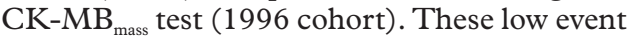
rates were accomplished using a carefully applied protocol which is integrated into everyday clinical routine in our cardiac emergency room. The protocol requires the time of onset of symptoms to be estimated as accurately as possible in patients presenting with chest pain. The subsequent timing of blood samples was performed relative to the time of onset of symptoms. In addition, minor myocardial damage was detected using the $\mathrm{CK}-\mathrm{MB}_{\text {mass }}$ critical difference: serial $\mathrm{CK}-\mathrm{MB}_{\text {mass }}$ values were considered abnormal when the difference between two serial samples exceeded the reference change value. ${ }^{13}$ Moreover, patients stayed for observation for a maximum of 12 hours after the onset of symptoms (in 1994 the maximum was 24 hours) and they were discharged after an uneventful observation period and after ruling out myocardial infarction. The observation period of 12 hours was chosen when it was shown by Lee and colleagues that a 12 hour strategy was safe for patients at low risk. ${ }^{18}$ Using our carefully defined sampling protocol, the event rate after discharge was very low, and was comparable with the event rate reported by Hamm and colleagues. ${ }^{9}$ In addition, we have extended our observation to a six month and a 24 month follow up, which showed a low incidence of adverse events over a two year period.

Although the troponins ( $\mathrm{T}$ and $\mathrm{I}$ ) are increasingly used for risk stratification in patients with acute coronary syndromes, there is not much evidence at present that they will make a major contribution to present clinical practice with respect to ruling out myocardial infarction. In contrast, a recent paper by Pope and colleagues on missed diagnosis of acute myocardial ischaemia in the emergency department showed a very low percentage of patients inadvertently discharged $(2.1-2.3 \%)$ using a rule-out myocardial infarction protocol that included serial CK-MB measurements but no troponin measurements. ${ }^{38}$ Whether a troponin $\mathrm{T}$ or I measurement added to such a protocol would substantially improve clinical outcome in these patients remains to be demonstrated.

LIMITATIONS OF THE STUDY

This study has some limitations. The 1996 cohort was nearly twice as large as the 1994 cohort, whereas the total number of patients presenting with chest pain at the cardiac emergency room in both cohorts was similar (1162 in 1996 and 1109 in 1994), and the number of patients with chest pain who were admitted to the coronary care unit was also similar (398v 393). This may reflect a more aggressive discharge policy combined with the utilisation of serial CK-MB sampling in a larger proportion of patients in 1996.

Some baseline characteristics differed between the two cohorts. These data were obtained from the database, from medical records, and from the patients or the patients' relatives. The differences may reflect a change in the patient population over the years, or a different admission policy, with more beds being available in 1996 because of a reduction in the length of stay. However, although the proportion of patients with a previous myocardial infarct was lower in the 1996 cohort than in the 1994 cohort, the incidence of a history of diabetes mellitus and hypertension was higher, indicating that the 1996 cohort was not a lower risk group than the 1994 cohort. Our study was of course not randomised, and there could have been changes in the use of diagnostic tests and refinements of treatment between 1994 and 1996 (applied in the outpatient department, 
not in the cardiac emergency room), which remained undetected but which might have influenced our results.

CONCLUSIONS

Our data show that, using a rule-out protocol for myocardial infarction with a rapid and sensitive $\mathrm{CK}-\mathrm{MB}_{\text {mass }}$ assay and serial sampling, the length of stay of patients with chest pain in the cardiac emergency room was significantly reduced by $44 \%$ - that is, from a median of 16.0 hours to a median of 9.0 hours, compared with the protocol used up to 1994 . These patients were discharged home from the cardiac emergency room without compromising safety - the incidence of adverse events was low during the subsequent 30 days, six months, and 24 months.

1 Goldman L, Cook EF, Brand DA, et al. A computer protocol to predict myocardial infarction in emergency department patients with chest pain. N Engl f Med 1988;318:797803.

2 Polanczyk CA, Johnson PA, Cook EF, et al. A proposed strategy for utilization of creatine kinase-MB and troponin I in the evaluation of acute chest pain. Am $\mathcal{F}$ Cardiol 1999 83:1175-9.

3 Zimmerman J, Fromm R, Meyer D, et al. Diagnostic marker cooperative study for the diagnosis of myocardial infarction. Circulation 1999;99:1671-7.

4 De Winter RJ, Koster RW, Sturk A, et al. Value of myoglobin, troponin T, and CK-MB mass in ruling out an myoglobin, troponin T, and Cocardial infarction in the emergency room. Circulation 1995;92:3401-7.

5 Johnson PA, Goldman L, Sacks DB, et al. Cardiac troponin $\mathrm{T}$ as a marker for myocardial ischemia in patients seen at T as a marker for myocardial ischemia in patients seen at the emergency depart

6 De Winter RJ, Lijmer JG, Koster RW, et al. Diagnostic accuracy of myoglobin concentration for the early diagnosis of acute myocardial infarction. Ann Emerg Med 2000;35:113-20

7 Fesmire FM, Percy RF, Bardoner JB, et al. Serial creatinine kinase $(\mathrm{CK}) \mathrm{MB}$ testing during the emergency departmen evaluation of chest pain: utility of a 2-hour delta CK-MB of $+1.6 \mathrm{ng} / \mathrm{ml}$. Am Heart f 1998;136:237-44.

8 De Winter RJ, Bholasingh R, Nieuwenhuijs AB, et al. Ruling out acute myocardial infarction early with two serial creatine kinase-MBmass determinations. Eur Heart 7 1999;20: 967-72.

9 Hamm CW, Goldmann BU, Heeschen C, et al. Emergency room triage of patients with acute chest pain by means of
rapid testing for cardiac troponin T or troponin I. N Engl $\mathcal{F}$ Med 1997;337:1648-53.

10 Collinson PO, Rosalki SB, Kuwana T, et al. Early diagnosis of acute myocardial infarction by CK-MB mass measureof acute myocardial infarction by CK-MB
ments. Ann Clin Biochem 1992;29:43-7.

11 Hedges JR, Gibler WB, Young GP, et al. Multicenter study of creatine kinase-MB use: effect on chest pain clinical decision making. Acad Emerg Med. 1996;3:7-15

12 Mair J, Artner-Dworzak E, Dienstl A, et al. Early detection of acute myocardial infarction by measurement of mas concentration of creatine kinase-MB. $A m \mathcal{f}$ Cardiol 1991;68:1545-50

13 De Winter RJ, Koster RW, Gorgels JP, et al. Critical difference between serial measurements of CK-MB mass to detect myocardial damage. Clin Chem 1997;43:338-43.

14 World Health Organisation. Criteria for the diagnosis of acute myocardial infarction. Proposal for the multinational monitoring of trends and determinants in cardiovascular disease. Geneva: Cardiovascular Disease Unit of WHO, 1981.

15 Fiolet JW, Willebrands AF, Lie KI, et al. Determination of creatine kinase isoenzyme $\mathrm{MB}(\mathrm{CK}-\mathrm{MB})$ : comparison of methods and clinical evaluation. Clin Chim Acta 1977;80: 23-35.

16 Mercer DW. Immunochemical determination of LD-1 and LD-2 in CK-MB fraction of column eluates. Clin Chem 1981;27:779-80

17 Ross SM, Fraser CG. Biological variation of cardiac markers: analytical and clinical considerations. Ann Clin Biochem 1998;35:80-4.
18 Lee TH, Juarez G, Cook EF, et al. Ruling out acute myocardial infarction. A prospective multicenter validation of a 12-hour strategy for patients at low risk. $N \mathrm{Engl} \mathrm{F} \mathrm{Med}$ 1991;324:1239-46.

19 Zalenski RJ, Rydman RJ, McCarren M, et al. Feasibility of a rapid diagnostic protocol for an emergency department chest pain unit. Ann Emerg Med 1997;29:99-108.

20 Lee TH, Cook EF, Weisberg M, et al. Acute chest pain in the emergency room. Identification and examination of lowrisk patients. Arch Intern Med 1985;145:65-9.

21 Gomez MA, Anderson JL, Karagounis LA, et al. An emergency department-based protocol for rapidly ruling out myocardial ischemia reduces hospital time and expense: results of a randomized study (ROMIO). $7 \mathrm{Am}$ Coll Cardiol 1996;28:25-33.

22 Goldman L, Cook EF, Johnson PA, et al. Prediction of the need for intensive care in patients who come to the emergency departments with acute chest pain. $N$ Engl f Med 1996;334:1498-504.

23 Gibler WB, Runyon JP, Levy RC, et al. A rapid diagnostic and treatment center for patients with chest pain in the emergency department. Ann Emerg Med 1995;25:1-8.

24 Green GB, Beaudreau RW, Chan DW, et al. Use of troponin $\mathrm{T}$ and creatine kinase-MB subunit levels for risk stratification of emergency department patients with possible myocardial ischemia. Ann Emerg Med 1998;31:19-29.

25 Selker HP, Zalenski RJ, Antman EM, et al. An evaluation of technologies for identifying acute cardiac ischemia in the emergency department: a report from a National Heart Attack Alert Program Working Group. Ann Emerg Med 1997;29:13-87.

26 Aufderheide TP, Rowlandson I, Lawrence SW, et al. Test of the acute cardiac ischemia time-insensitive predictive instrument (ACI-TIPI) for prehospital use. Ann Emerg Med 1996;27:193-8.

27 Tatum JL, Jesse RL, Kontos MC, et al. Comprehensive strategy for the evaluation and triage of the chest pain patient. Ann Emerg Med 1997;29:116-25.

28 Selker HP, Zalenski RJ, Antman EM, et al. An evaluation of technologies for identifying acute cardiac ischemia in the emergency department: executive summary of a National Heart Attack Alert Program Working Group Report. Ann Emerg Med 1997;29:1-12.

29 Wu AH, Apple FS, Gibler WB, et al. National Academy of Clinical Biochemistry Standards of Laboratory Practice: recommendations for the use of cardiac markers in coronary artery diseases. Clin Chem 1999;45:1104-21.

30 Panteghini M. IFCC Committee on Standardization of Markers of Cardiac Damage: premises and project presentation. International Federation of Clinical Chemistry and Laboratory Medicine. Clin Chem Lab Med 1998;36:88793.

31 Collinson PO, Ramhamadamy EM, Stubbs PJ, et al. Rapid enzyme diagnosis of patients with acute chest pain reduces patient stay in the coronary care unit. Ann Clin Biochem 1993;30:17-22

$32 \mathrm{Wu} \mathrm{AH}$, Clive JM. Impact of CK-MB testing policies on hospital length of stay and laboratory costs for patients with myocardial infarction or chest pain. Clin Chem 1997;43: 326-32.

33 Anderson FP, Jesse RL, Nicholson CS, et al. The costs and effectiveness of a rapid diagnostic and treatment protocol for myocardial infarction. In: Bowie LJ, ed. Assessing clinical outcomes. Utilizing appropriate laboratory testing to decrease healthcare costs and improve patient outcomes (American Association of Clinical Chemistry Leadership Series). Washington, DC: American Association of Clinical Chemistry, 1996:20-4.

34 Apple FS, Preese LM, Riley L, et al. Financial impact of a rapid CK-MB-specific immunoassay on the diagnosis of myocardial infarction. Arch Pathol Lab Med 1990;114: 1017-20.

35 Polanczyk CA, Lee TH, Cook EF, et al. Value of additional two-hour myoglobin for the diagnosis of myocardial infarction in the emergency department. Am f Cardiol 1999;83: $525-9$.

36 Hamilton RW, Hopkins MB, Shihabi ZK. Myoglobinuria, hemoglobinuria, and acute renal failure. Clin Chem 1989;35:1713-20.

37 Ravkilde $\mathrm{J}$, Nissen $\mathrm{H}$, Horder $\mathrm{M}$, et al. Independent prognostic value of serum creatine kinase isoenzyme MB mass, cardiac troponin $\mathrm{T}$ and myosin light chain levels in suspected acute myocardial infarction. Analysis of 28 months of follow-up in 196 patients. $\mathcal{f} \mathrm{Am}$ Coll Cardiol months of follow

38 Pope JH, Auferheide TP, Ruthazer R, et al. Missed diagnoses of acute cardiac ischemia in the emergency department. N Engl f Med 2000;342:1163-70. 